\title{
Validation of a Stability-indicating RP-LC Method for the Assessment of Recombinant Human Interleukin-11 and Its Correlation with Bioassay
}

\author{
Ricardo Bizogne Souto, * Fernanda Pavani Stamm,* Maria Teresa de Carvalho Pinto Ribela, ** \\ Paolo Bartolini,** Guilherme Zanini Calegari,* and Sérgio Luiz Dalmora** \\ *Department of Industrial Pharmacy, Federal University of Santa Maria, 97105-900 Santa Maria-RS, Brazil \\ **Biotechnology Department, IPEN-CNEN, Cidade Universitária, 05508-900 São Paulo-SP, Brazil
}

\begin{abstract}
A stability-indicating reversed-phase liquid chromatography (RP-LC) method was validated for the assessment of recombinant human interleukin-11 (rhIL-11), based on the ICH guidelines. The method was carried out on a Jupiter $\mathrm{C}_{4}$ column $\left(250 \mathrm{~mm} \times 4.6 \mathrm{~mm}\right.$ i.d.), maintained at $25^{\circ} \mathrm{C}$. The mobile phase A consisted of $0.1 \%$ trifluoroacetic acid (TFA) and the mobile phase B was acetonitrile with $0.1 \%$ TFA, run at a flow rate of $1 \mathrm{~mL} / \mathrm{min}$, and using a photodiode array (PDA) detection at $214 \mathrm{~nm}$. Separation was obtained with a retention time of $27.6 \mathrm{~min}$, and was linear over the concentration range of $1-200 \mu \mathrm{g} / \mathrm{mL}\left(r^{2}=0.9995\right)$. Specificity was established in degradation studies, which also showed that there was no interference of the excipients. The accuracy was $100.22 \%$ with bias lower than $1.25 \%$. Moreover, the in vitro cytotoxicity test of the degraded products showed non-significant differences $(p>0.05)$. The method was applied to the assessment of rhIL-11 and related proteins in biopharmaceutical dosage forms, and the results were correlated to those of a bioassay.
\end{abstract}

(Received November 11, 2011; Accepted December 6, 2011; Published March 10, 2012)

\section{Introduction}

Interleukin 11 (IL-11) is a multifunctional cytokine in the IL-6 type subfamily of long-chain helical cytokines, which modulates the proliferation, differentiation and maturation of various types of hematopoietic cells. Recombinant human interleukin-11 (rhIL-11) produced by DNA technology in Escherichia coli is currently being used worldwide for the prevention of severe chemotherapy-induced thrombocytopenia and to reduce the need for platelet transfusions in patients with nonmyeloid malignancies. Further anti-inflammatory applications of rhIL-11 include chemotherapy-induced oral mucositis, treatments of Crohn's disease, rheumatoid arthritis, protection of intestinal epithelial cells from tissue damage and attenuation of cardiac fibrosis after myocardial infarction. ${ }^{1-3}$

The polypeptide structure of rhIL-11 contains 177 amino acids with a molecular mass of $19 \mathrm{kDa}$ and differs from the naturally occurring protein only by the absence of the amino-terminal proline, and the presence of two residues of $\mathrm{Met}^{58}$ and $\mathrm{Met}^{122}{ }^{4}$

The biological potency of rhIL-11 has been assessed by the in vitro cell culture-based bioassay using T10 cells derived from the T1165 murine plasmacytoma line. ${ }^{5}$ The bioassay was used to evaluate the effect on the bioactivity of chemical modifications combined with site-directed and deletion mutagenesis and to identify functionally critical regions/residues of the biomolecule. $^{4,6}$ Also, early signals triggered by IL-11 were

$\dagger$ To whom correspondence should be addressed.

E-mail: sdalmora@terra.com.br assessed in a multifactor-dependent human erythroleukemic cell line, TF1, which showed that this protein stimulated cell proliferation, induced a protein tyrosine phosphorylation, and activated proto-oncogene expression in the cell line. ${ }^{7}$ The bioactivity of recombinant truncated human interleukin-11 expressed as a fusion protein in E. coli was also evaluated by the in vitro proliferation assay using the 7TD1 cell line. ${ }^{8}$

The kinetics of megakaryopoiesis induced by rhIL-11 was analyzed in myelo-suppressed mice after intravenous injection of $40 \mu \mathrm{g}$ of mitomycin $\mathrm{C} / 20 \mathrm{~g}$ and treatment with a dose of $10 \mu \mathrm{g} / 20 \mathrm{~g} /$ day for 21 consecutive days. The results suggested that rhIL-11 ameliorates thrombocytopenia via the stimulation of both maturation and commitment, followed by the proliferation of megakaryocytic cells. ${ }^{9}$

Many analytical techniques are available to monitor the purity, the chemical stability, and the potency of biopharmaceutical proteins obtained through recombinant technology. Reversed-phase liquid chromatography (RP-LC) offers a high level of accuracy and sensitivity for the analysis of closely related protein variants or degradation products which may have reduced activity and altered immunogenicity. ${ }^{10,11}$ One drawback of this technique is that proteins are usually denatured or dissociated as they are adsorbed on to the column matrix, and, therefore the technique is not appropriate for use as a single technique for establishing the potency of preparations that contain biologically inactive, non-covalent oligomers and polymers. Chemical modification approaches, combined with site-directed and deletion mutagenesis performed to identify functionally critical regions/residues of rhIL-11, were analyzed by gradient RP-LC using a $\mathrm{C}_{18}$ column with detection at 214 and $280 \mathrm{~nm} .{ }^{4}$ A RP-LC using a $\mathrm{C}_{4}$ column was used to separate 
and quantify rhIL-11 fusion protein expressed in E. coli and also for degradation kinetics studies. ${ }^{12,13}$ A linear gradient RP-LC method using $\mathrm{C}_{4}$ column and UV detection at $214 \mathrm{~nm}$ was employed to determine the oxidative effect of disposable laboratory plastic tubes used for storage of rhIL-11 samples. ${ }^{14}$ Also, a RP-LC method has been applied together with electrospray ionization mass spectrometry and capillary electrophoresis for the characterization of isomeric and deamidated forms. ${ }^{6}$ Optimal storage stability of lyophilized rhIL-11 was evaluated by quantifying the oxidation levels and cleavage products by gradient RP-LC using a Poros R1/H column with detection at $214 \mathrm{~nm} .{ }^{15}$ Mass spectrometry approaches have been used to identify proteins, namely peptide mass mapping and characterize the sequence of peptides. ${ }^{16}$ Physicochemical techniques cannot yet predict the biological activity, but can produce information about structure and composition and are used to monitor content, purity, and chemical stability of the biotechnology-derived products. No single technique can satisfactorily provide sufficient information about the protein. Thus, a combination of physico-chemical, immunological, and biological methods has been successfully applied in correlation studies between physic-chemical and biological assays, in attempts to find methods for characterization and for monitoring the stability of different biotherapeutics. ${ }^{17-20}$ At the moment, rhIL-11 is not included in any Pharmacopoeia and there are no validated methods for quality control analysis, as recommended by $\mathrm{ICH}$ guidelines. ${ }^{21}$ Besides, the biotherapeutic should meet the specifications and acceptance criteria for biotechnology-derived proteins..$^{22}$

The aim of the this research was to develop and validate a specific, sensitive and stability-indicating gradient RP-LC method for the content/potency assessment of rhIL-11; we want to correlate the results to the bioassay, and to evaluate the bioactivity and the cytotoxicity of the related proteins, thus contributing to the development of methods to monitor stability during the biotechnology steps, improve quality control, and thereby assure the therapeutic efficacy of the biological medicine.

\section{Experimental}

\section{Chemicals and reagents}

The reference reagent interleukin-11, human rDNA derived, WHO 92/788, was obtained from the National Institute for Biological Standards and Control-NIBSC (Herts, UK). The biological reference substance of rhIL-11 (BRS-rhIL-11), for physicochemical assays, was supplied by Amoytop Biotech Co., Ltd. (Xiamen, Fujian, China). A total of six batches of Plaquemax $^{\circledR}$ (Bergamo, São Paulo, Brazil), containing $5 \mathrm{mg} / \mathrm{vial}$ of rhIL-11 were identified by numbers from 1 to 6 and two batches of Neumega ${ }^{\circledR}$ (Wyeth, São Paulo, Brazil), containing $5 \mathrm{mg} / \mathrm{vial}$ of rhIL-11 were identified by numbers from 7 to 8 . The samples were obtained from commercial sources within their shelf life period. Hydrogen peroxide (30\%) in aqueous solution, potassium phosphate monobasic, polyssorbate 80 , acetonitrile and trifluoroacetic acid (TFA) were purchased from Merck (Darmstadt, Germany). All chemicals used were of pharmaceutical or special analytical grade. For all of the analyses, ultrapure water was obtained using an Elix 3 coupled to a Milli-Q Gradient A10 system Millipore (Bedford, MA). All solutions were degassed by ultrasonication using Tecnal (São Paulo, SP, Brazil) and filtered through a $0.22-\mu \mathrm{m}$ Millex filter Millipore (Bedford, MA).

\section{Apparatus}

The LC method was carried out on a Shimadzu LC system (Shimadzu, Kyoto, Japan) equipped with an SCL-10Avp system controller, an LC-10 AD $\mathrm{vp}$ pump, a DGU-14A degasser, an SIL-10AD ${ }_{\mathrm{VP}}$ autosampler, and an SPD-M10AVP PDA detector. The peak areas were integrated automatically by computer using a Shimadzu Class VP ${ }^{\circledR}$ V 6.14 software program.

\section{Samples and standard solutions}

Working standard and sample solutions were prepared daily by diluting the BRS-rhIL-11 and the samples of pharmaceutical formulations in $0.05 \mathrm{M}$ phosphate buffer ( $\mathrm{pH} 7.4$ ) containing $0.5 \mathrm{mg} / \mathrm{mL}$ of polysorbate 80 , to a final concentration of $50 \mu \mathrm{g} / \mathrm{mL}$.

\section{Procedures}

Reversed-phase liquid chromatography (RP-LC). The experiments were performed on a reversed-phase Phenomenex (Torrance, USA) Jupiter $\mathrm{C}_{4}$ column $(250 \mathrm{~mm} \times 4.6 \mathrm{~mm}$ i.d., with a pore size of $300 \AA$ ) maintained at $25^{\circ} \mathrm{C}$. A security guard holder was used to protect the analytical column. The elution was performed using a linear gradient at a constant flow rate of $1 \mathrm{~mL} / \mathrm{min}$, with photodiode array (PDA) detection at $214 \mathrm{~nm}$. Mobile phase A consisted of $0.1 \%$ TFA and mobile phase B was acetonitrile, with $0.1 \%$ TFA, run as follows: time 0 to $0.1 \mathrm{~min}$ $40 \%$ of $\mathrm{B}$; from 0.1 to 30 min linear up to $65 \%$ of $\mathrm{B}$; from 30.01 to 31 min linear down to $40 \%$ of $\mathrm{B}$, maintained up to $40 \mathrm{~min}$. The mobile phases were filtered through a $0.22-\mu \mathrm{m}$ membrane Millipore filter (Bedford, MA). The injection volume was $50 \mu \mathrm{L}$ of a solution containing $50 \mu \mathrm{g} / \mathrm{mL}$ for both the reference standard and the samples.

In vitro TF-1 cell proliferation bioassay. The assay was performed as described elsewhere, ${ }^{7,23}$ and the growth-promoting activity of rhIL-11 was assessed on a TF-1 cell line (ATCC number CRL-2003). The cells were maintained in culture medium RPMI 1640 containing 10\% (v/v) fetal bovine serum Sigma-Aldrich (St. Louis, MO); concentrations of GM-CSF $(1-20 \mathrm{ng} / \mathrm{mL})$ were added for cell proliferation in $75 \mathrm{~cm}^{2}$ flasks for $24 \mathrm{~h}$, seeding at approximately $2.0-6.0 \times 10^{5}$ cells $/ \mathrm{mL}$. The assay was performed in triplicate; the cells were seeded in 96-well BD Biosciences microplates (San Jose, CA) at a density of $4 \times 10^{5}$ cells $/ \mathrm{mL}\left(2 \times 10^{4}\right.$ cells/well $)$ and dosed on seeding with a two-fold dilution series range starting with $65 \mathrm{IU} / \mathrm{mL}$ $(250 \mu \mathrm{g} / \mathrm{mL})$ of rhIL-11. The WHO reference reagent rhIL-11 (92/788) was used as standard and the negative control was RPMI 1640 culture medium. Briefly, the plates were incubated at $37^{\circ} \mathrm{C}$ in $5 \% \mathrm{CO}_{2}$ for a minimum of $24 \mathrm{~h}$. Then $25 \mu \mathrm{L} /$ well of MTT solution $(5 \mathrm{mg} / \mathrm{mL})$ was added and the plates were incubated for a further $5 \mathrm{~h}$. Following the addition of $100 \mu \mathrm{L} /$ well of sodium dodecyl sulfate $(240 \mathrm{mg} / \mathrm{mL})$ overnight, the absorbance was assessed at $595 \mathrm{~nm}$, using a microplate reader Thermo Scientific Multiskan FC (Vantaa, Finland).

In vivo thrombocytopenic bioassay. Female 7 to 8 weeks-old $\mathrm{Balb} / \mathrm{c}$ mice weighing between 18 and $23 \mathrm{~g}$ were housed in air-conditioned, controlled conditions (room temperature $22 \pm 2^{\circ} \mathrm{C}$, relative humidity of $50-60 \%$, and artificial illumination, $12 \mathrm{~h}$ per day). They were given free access to food and water. All of the assays were conducted in accordance with the National Protection Laws on Animal Welfare.

The bioassay was performed as described elsewhere, ${ }^{9}$ and modified accordingly. The animals were allocated to sample, standard, and control groups in a fully randomized order and identified by color code for the assay, usually with 6 mice per treatment group. Standard and test samples were diluted to the concentrations of 350 and $700 \mu \mathrm{g} / \mathrm{mL}$ with phosphate-buffered 
saline, pH 7.2, containing $0.1 \%$ bovine serum albumin. Mice were given a dose of $40 \mu \mathrm{g}$ of mytomycin C/20 g/0.25 mL subcutaneously on days 0 and 1 to induce myelo-suppression, severe thrombocytopenia without any animal death. Multiple injections of $0.25 \mathrm{~mL}$ rhIL-11 per mouse were administered in to the mytomycin $C$ treated mice, from day 2 to day 19 . Twenty four hours after the last rhIL-11 injection, the mice were anaesthetized, and peripheral blood was collected from the inferior vena cava. Blood cell count analysis of platelets was performed using a Cell-Dyn ${ }^{\circledR} 3200$ (Abbott Laboratories, Saint-Laurent, Québec, Canada). The smears for the May-Grünwald-Giemsa method were prepared on glass slides and stained, then the white cells were counted and expressed as percentage of the total number of neutrophils.

In vitro cytotoxicity test. The in vitro cytotoxicity method was performed as described elsewhere, ${ }^{24}$ based on a neutral red uptake (NRU) assay, with the exposure of NCTC clone 929 cell line (mammalian fibroblasts, ATCC number CCL-1) to the degraded samples of rhIL-11. The $\mathrm{pH}$ of the samples was adjusted to 7.0, and positive and diluent controls were included in the assay together with BRS-rhIL-11 solution. The NRU assay was performed on 96-well microplates, maintained at $37^{\circ} \mathrm{C}$ in a $\mathrm{CO}_{2}$ incubator for $24 \mathrm{~h}$, with a cell suspension density of approximately $2 \times 10^{5}$ cells $/ \mathrm{mL}$. Neutral red release was evaluated by the addition of an extractant solution, and the absorbance was measured at $540 \mathrm{~nm}$.

Validation of reversed-phase liquid chromatography method. The method was validated using samples of a biopharmaceutical formulation of rhIL-11 with a label claim of $5 \mathrm{mg} / \mathrm{vial}$ by determinations of the following parameters: specificity, linearity, range, precision, accuracy, limit of detection (LOD), limit of quantitation (LOQ), robustness, stability, and system suitability test, following the ICH guidelines. ${ }^{21}$

Specificity. The specificity of the method for the biomolecule was assessed by determination of the peak purity of reference solutions and of the samples of biopharmaceutical formulation of rhIL-11 $(50 \mu \mathrm{g} / \mathrm{mL})$ previously degraded by hydrogen peroxide. In addition, the interference of excipients in the biopharmaceutical formulations was determined by injection of a sample containing only placebo (in-house mixture of formulation excipients). Additionally, the BRS-rhIL-11 and the degraded samples were subjected to the in vitro cytotoxicity test.

Linearity. The linearity was determined by constructing three independent analytical curves, each one with nine concentrations of the BRS-rhIL-11, over the range of $1-200 \mu \mathrm{g} / \mathrm{mL}$. Three replicate $50 \mu \mathrm{L}$ injections of the reference solutions were prepared to verify the repeatability of the detector response. The peak areas of the chromatograms were plotted against the respective concentrations of rhIL-11 to obtain the analytical curve. The results were subjected to regression analysis by the least squares method to calculate calibration equation and the determination coefficient.

Precision and accuracy. The precision of the method was determined by repeatability (intra-day) and intermediate precision (inter-days). Repeatability was examined by six evaluations of sample of rhIL-11, on the same day, under the same experimental conditions. The intermediate precision of the method was assessed by analyzing two samples of the pharmaceutical formulations on three different days (inter-days) and also by other analysts performing the analysis in the same laboratory (between-analysts). The accuracy was assessed applying the proposed method to the analysis of the in-house mixture of the excipients with known amounts of the biomolecule, to obtain solutions at concentrations of 40, 50 and
$60 \mu \mathrm{g} / \mathrm{mL}$, equivalent to 80,100 and $120 \%$ of the nominal analytical concentration, respectively. The accuracy was calculated as the percentage of the drug recovered from the formulation and also expressed as the percentage relative error (bias\%) between the measured mean concentrations and the added concentrations.

Limits of detection and quantitation. The limit of detection (LOD) and the limit of quantitation (LOQ) were calculated, as defined by $\mathrm{ICH},{ }^{21}$ by using the mean values of the three independent analytical curves determined by a linear-regression model, where the factors 3.3 and 10 for the detection and quantitation limits, respectively, were multiplied by the ratio of the standard deviation of the intercept and the slope. The LOQ was also evaluated in an experimental assay.

Robustness. The robustness of an analytical procedure refers to its ability to remain unaffected by small and deliberate variations in method parameters; it provides an indication of its reliability for the routine analysis. The robustness was determined by analyzing the same samples $(50 \mu \mathrm{g} / \mathrm{mL})$ under a variety of conditions of the method parameters, such as: injection volume, percent of acetonitrile, flow rate, temperature and the wavelength. To assess the stability of sample solutions of rhIL-11, we tested the samples after they were maintained at $2-8^{\circ} \mathrm{C}$ for $48 \mathrm{~h}$ and also placed into the auto sampler, at room temperature, for $24 \mathrm{~h}$. The stability of these solutions was studied by observing any change in the chromatographic pattern, compared with the pattern from freshly prepared solutions.

System suitability test. To ensure the validity of the analytical procedure, we used data from five injections of $50 \mu \mathrm{L}$ of the working BRS-rhIL-11 solution containing $50 \mu \mathrm{g} / \mathrm{mL}$ for evaluation of the system suitability parameters, such as asymmetry, number of theoretical plates, retention time, and peak area, through the CLASS-VP ${ }^{\circledR} \mathrm{V} 6.12$ software.

Analysis of rhIL-11 in biopharmaceutical formulations. The biopharmaceutical samples were previously identified by SDS-PAGE followed by transference to a nitrocellulose membrane and immunodetection with a rhIL-11-specific antiserum (Santa Cruz Biotechnology, Santa Cruz, CA). For the quantitation of rhIL-11 by the proposed method, the respective solutions were diluted to appropriate concentration with phosphate buffer containing $0.5 \mathrm{mg} / \mathrm{mL}$ of polysorbate 80 and injected in triplicate; the percentage recoveries of the biopharmaceutical materials were calculated against the BRS-rhIL-11.

\section{Results and Discussion}

\section{Optimization of chromatographic conditions}

To obtain the best chromatographic conditions, we optimized the mobile phase to provide appropriate selectivity and sensitivity. Phosphate buffered saline solution resulted in higher sensitivity compared to phosphate buffer and phosphoric acid solution. The use of a gradient flow-rate resulted in better sensitivity, improving the peak symmetry with the retention time also suitable for the separation of deamidated materials and sulfoxides, as previously suggested. ${ }^{10}$ The optimum wavelength was selected using the PDA detector. The optimized conditions of the LC method were validated for the analysis of rhIL-11 in biopharmaceutical formulations and correlation studies.

\section{Method validation}

Specificity. Specificity of the method for the biological materials was evaluated under oxidative conditions by adding 


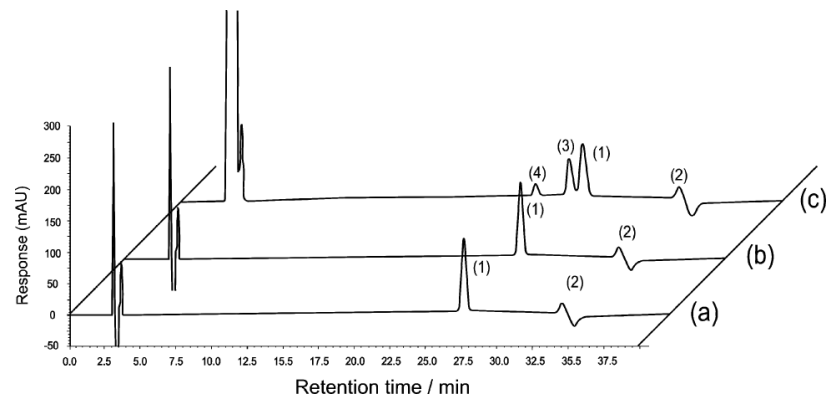

Fig. 1 RP-LC chromatograms of rhIL-11 $(50 \mu \mathrm{g} / \mathrm{mL})$

(a)

BRS-rhIL-11. Peak: 1, rhIL-11; 2, excipients. (b) Biopharmaceutical formulations. Peak: 1, rhIL-11; 2, excipients. (c) After degradation by oxidative conditions. Peak: 1, rhIL-11; 2, excipients; 3, sulfoxides; 4, deamidated.

Table 1 Inter-days and between-analysts precision data of RP-LC for rhIL-11 in biopharmaceutical formulations

\begin{tabular}{|c|c|c|c|c|c|c|}
\hline \multicolumn{4}{|c|}{ Inter-day } & \multicolumn{3}{|c|}{ Between-analysts } \\
\hline Sample & Day & $\begin{array}{c}\text { Recovery } \\
\%\end{array}$ & $\begin{array}{c}\mathrm{RSD}^{\mathrm{b}}, \\
\%\end{array}$ & Analyst & $\begin{array}{c}\text { Recovery } \\
\%\end{array}$ & $\begin{array}{c}\mathrm{RSD}^{\mathrm{b}}, \\
\%\end{array}$ \\
\hline \multirow[t]{3}{*}{1} & 1 & 102.81 & 1.78 & A & 100.85 & 1.32 \\
\hline & 2 & 100.41 & & B & 103.42 & \\
\hline & 3 & 99.29 & & $\mathrm{C}$ & 101.41 & \\
\hline \multirow[t]{3}{*}{2} & 1 & 100.51 & 1.67 & A & 98.76 & 1.63 \\
\hline & 2 & 102.63 & & B & 100.71 & \\
\hline & 3 & 99.31 & & $\mathrm{C}$ & 102.01 & \\
\hline
\end{tabular}

a. Mean of three replicates.

b. RSD, relative standard deviation.

$50 \mu \mathrm{L}$ of hydrogen peroxide $3 \%$ for 3 min, generating the main peak at $27.6 \mathrm{~min}$ and additional peaks of related proteins at retention times 24.2 and $26.5 \mathrm{~min}$, respectively, as shown in the typical chromatograms in Fig. 1. Moreover, the interference of the excipients of the biopharmaceutical formulation was determined by the injection of a sample containing only a placebo (in-house mixture of the formulation excipients), giving no additional peak. Then the specificity of the method was established by determining the peak purity in the samples using a PDA detector. It is possible that other physical or chemical factors may affect the stability of the protein and might produce degraded products which are not detected by the RP-LC method. Additionally the BRS-rhIL-11 and the degraded samples were subjected to the in vitro cytotoxicity test.

Linearity. The analytical curves constructed for rhIL-11 were found to be linear in the $1-200 \mu \mathrm{g} / \mathrm{mL}$ range. The value of the determination coefficient calculated $\left(r^{2}=0.9995, y=(42507 \pm\right.$ $178.242) x+(38447 \pm 4767.489)$, where, $x$ is concentration and $y$ is the peak absolute area) indicated linearity of the analytical curve for the method.

Precision. The precision of the method was studied by calculating the relative standard deviation (RSD\%) for six analyses at a concentration of $50 \mu \mathrm{g} / \mathrm{mL}$, performed on the same day and under the same experimental conditions. The obtained RSD value was $0.91 \%$. The intermediate precision was assessed by analyzing two samples of the pharmaceutical formulation on three different days (inter-days), giving RSD values of 1.67 and $1.78 \%$, respectively. The between-analysts precision was determined by calculating the RSD for the analysis of two
Table 2 Accuracy of RP-LC for rhIL-11 in the formulations

\begin{tabular}{ccrrr}
\hline $\begin{array}{c}\text { Nominal } \\
\begin{array}{c}\text { concentration/ } \\
\mu \mathrm{g} \mathrm{mL}^{-1}\end{array}\end{array}$ & $\begin{array}{c}\text { Mean concentration } \\
\text { measured }^{\mathrm{a}} /\end{array}$ & $\begin{array}{c}\mathrm{RSD}^{\mathrm{b}}, \\
\mu \mathrm{g} \mathrm{mL}^{-1}\end{array}$ & $\begin{array}{c}\text { Accuracy, } \\
\%\end{array}$ & $\begin{array}{c}\text { Bias }^{\mathrm{c}}, \\
\%\end{array}$ \\
\hline 40 & 40.06 & 1.34 & 100.15 & 0.15 \\
50 & 50.62 & 0.59 & 101.25 & 1.25 \\
60 & 59.56 & 0.56 & 99.26 & 0.74 \\
\hline
\end{tabular}

a. mean of three replicates.

b. RSD, relative standard deviation.

c. Bias, [(measured concentration - nominal concentration)/nominal concentration] $\times 100$.

Table 3 Chromatographic conditions and range investigated during robustness testing

\begin{tabular}{lcccc}
\hline \multicolumn{1}{c}{ Variable } & $\begin{array}{c}\text { Range } \\
\text { investigated }\end{array}$ & $\begin{array}{c}\text { rhIL-11 } \\
\%\end{array}$ & $\begin{array}{c}\mathrm{RSD}^{\mathrm{b}}, \\
\%\end{array}$ & $\begin{array}{c}\text { Optimized } \\
\text { value }\end{array}$ \\
\hline Flow rate/ & 0.9 & 101.59 & 0.50 & 1 \\
$\mathrm{~mL} \mathrm{~min}^{-1}$ & 1 & 100.58 & 0.34 & \\
& 1.1 & 99.91 & 043 & \\
Injection volume/ & 40 & 103.21 & 092 & 50 \\
$\mu \mathrm{L}$ & 50 & 99.39 & 0.38 & \\
& 60 & 101.58 & 0.53 & \\
Temperature $/{ }^{\circ} \mathrm{C}$ & 25 & 99.03 & 0.32 & 25 \\
& 30 & 100.78 & 0.58 & \\
Acetonitrile, $\%$ & 35 & 98.43 & 0.69 & \\
& 38 & 100.76 & 0.68 & 40 \\
Solution stability & 40 & 100.43 & 0.41 & \\
& Autosampler & 98.39 & 0.76 & \\
& $24 \mathrm{~h}$ & & 0.18 & - \\
& $2-8^{\circ} \mathrm{C}, 24 \mathrm{~h}$ & 98.58 & 1.47 & - \\
Wavelength/nm & $2-8^{\circ} \mathrm{C}, 48 \mathrm{~h}$ & 97.14 & 0.40 & - \\
\hline & $210-320$ & - & - & 214 \\
\hline
\end{tabular}

a. Mean of three replicates.

b. RSD, relative standard deviation.

samples of the biopharmaceutical formulations by three analysts; the values were found to be 1.32 and $1.63 \%$, respectively, as given in Table 1.

Accuracy. The accuracy was assessed from three replicate determinations of three solutions containing 40,50 and $60 \mu \mathrm{g} / \mathrm{mL}$, respectively. The absolute means obtained, with a mean value of $100.22 \%$ and a bias lower than $1.25 \%$ (Table 2), show that the method is accurate within the desired range.

Limits of detection and quantitation. The LOD and LOQ were calculated from the slope and the standard deviation of the intercept determined by a linear-regression model, by using the mean values of the three independent calibration curves. The obtained values were 0.34 and $1.12 \mu \mathrm{g} / \mathrm{mL}$, respectively. The evaluated experimental LOQ with a precision lower than $5 \%$ and accuracy within $\pm 5 \%,{ }^{25}$ was found to be $1 \mu \mathrm{g} / \mathrm{mL}$, and such a value was suitable for quality control analysis.

Robustness. The results and the experimental range of the selected variables evaluated are given in Table 3, together with the optimized values. There were no significant changes in the chromatographic pattern when modifications were introduced into the experimental conditions, thus showing the method to be robust. The stability of the sample solutions was studied; the data obtained showed the stability for $24 \mathrm{~h}$ in an auto sampler and for $48 \mathrm{~h}$ when maintained at $2-8^{\circ} \mathrm{C}$.

System suitability. The system suitability test was carried out to 
Table 4 Comparative content/potencies evaluation of rhIL-11 in biopharmaceutical formulations, by RP-LC method and TF-1 proliferation bioassay

\begin{tabular}{crcrcc}
\hline & \multicolumn{2}{c}{ RP-LC $^{\text {a }}$} & & \multicolumn{2}{c}{ In vitro bioassay } \\
\cline { 2 - 3 } \cline { 5 - 6 } Sample & $\begin{array}{c}\text { Main peak, } \\
\%\end{array}$ & $\begin{array}{c}\text { Deamidated/ } \\
\text { sulfoxides, } \%\end{array}$ & & $\begin{array}{c}\text { Potency, } \\
\%\end{array}$ & $\begin{array}{c}\text { Confidence interval } \\
(P=0.95)\end{array}$ \\
\hline 1 & 97.69 & 0.25 & & 95.04 & $88.15-107.12$ \\
2 & 101.17 & 1.16 & & 97.89 & $94.11-106.07$ \\
3 & 98.09 & 0.88 & & 100.14 & $95.66-109.31$ \\
4 & 97.60 & 0.56 & & 93.23 & $85.74-102.21$ \\
5 & 102.40 & 1.63 & & 103.30 & $96.14-110.98$ \\
6 & 101.36 & 1.57 & & 96.45 & $92.15-105.92$ \\
7 & 96.95 & 0.79 & & 93.26 & $85.16-101.17$ \\
8 & 103.83 & 0.34 & & 98.92 & $93.67-111.43$ \\
Mean & 99.88 & 0.77 & & 97.28 & - \\
SD $^{\mathrm{b}}$ & 2.60 & 0.43 & & 3.68 & - \\
\hline
\end{tabular}

a. Mean of three replicates.

b. SD, standard deviation.

evaluate the resolution and repeatability of the system for the analysis to be performed. The obtained RSD values for the retention time, peak symmetry and peak area were $1.19,0.73$ and $0.56 \%$, respectively. The number of theoretical plates was about 35673 , with RSD of $0.57 \%$. The parameters tested were within an acceptable range ( RSD $<2.0 \%$ ).

\section{Method application}

The validated RP-LC method was applied for the determination of rhIL-11 in biopharmaceutical formulations, giving content/potencies within 96.95 and $103.83 \%$ of the stated potency, with calculated RSD lower than $0.87 \%$, as shown in Table 4. The results demonstrated the stability-indicating capability and the application of the method, which could be also applied to support biosimilarity studies of biomolecules. ${ }^{26}$

\section{Biological potency assessment}

The potency of rhIL-11 was assessed by the TF-1 cell proliferation bioassay based on the dose-dependent growth curve of the cells, measuring the responses with MTT. The statistical analysis of the assay data was performed by a parallel line method, using PLA 2.0 Software (Stegmann System-beratung, Rodgau, Germany), which gave the values shown in Table 4. In addition, the biological activity of three biopharmaceutical samples was assessed by the in vivo thrombocytopenic bioassay, analyzing the results of peripheral platelet counts and of neutrophils number, respectively, giving a difference lower than $3.82 \%$, compared to the in vitro TF-1 cell proliferation bioassay. The biological model was previously used to evaluate the effects on the peripheral platelet counts of rhIL-11 chemically modified with hyaluronic acid and polyethylene glycol after injections in rats and in mice. ${ }^{27}$

Samples were also artificially degraded, analyzed by the RP-LC, and subjected to the in vitro and to the in vivo bioassays to evaluate the bioactivity of the sulfoxides/deamidated forms, non-detecting significant changes related of the intact rhIL-11. Previous studies by site-directed and deletion mutagenesis showed 3- to 25-fold decrease of the in vitro biological activity. ${ }^{4}$

\section{Cytotoxicity evaluation}

The cytotoxicity test was performed on degraded forms versus the intact molecule, in order to detect possible effects resulting from the instability of the samples during storage. However, this showed non-significant differences $(p>0.05)$. Such evaluations are now necessary, mainly due to the recent concerns related to possible human undesirable effects of the degraded forms. ${ }^{28}$

\section{Conclusion}

The results of the validation studies show that the RP-LC method is specific, sensitive with a LOQ of $1.0 \mu \mathrm{g} / \mathrm{mL}$ and accurate and that it possesses significant linearity and precision characteristics. Separation of rhIL-11 was achieved with a retention time of $27.6 \mathrm{~min}$, and the method was successfully used for the analysis of biopharmaceutical formulations, showing a higher mean difference of the estimated content/potencies of $2.60 \%$ compared to the in vitro TF- 1 cell proliferation bioassay, but with non-significant difference, as calculated by the Student's $t$-test $(p>0.05)$. This is the first published validated analytical method that meet ICH guidelines, and represents an improvement over current methods. This method can be applied in combination with the in vitro TF-1 cell proliferation bioassay, and with the expected development of a size-exclusion chromatographic method, to the characterization of rhIL-11 by monitoring its stability during the biotechnology process and, through subsequent purification steps, and to assure the batch-to-batch consistency of the bulk and finished biological products.

\section{Acknowledgements}

The authors wish to thank $\mathrm{CNPq}$ (Conselho Nacional de Desenvolvimento Científico e Tecnológico) Project 304860/2008-5, for financial support.

\section{References}

1. M. Ellis, U. Hedstrom, C. Frampton, H. Alizadeh, J. Kristensen, F. V. Shammas, and B. K. al-Ramadi, Clin. Immunol., 2006, 120, 129.

2. K. Usuki, A. Urabe, Y. Ikeda, Y. Ohashi, H. Mizoguchi, and F. Takaku, Int. J. Hematol., 2007, 85, 159.

3. S. Yanaka, E. Sano, N. Naruse, K. Miura, M. Futatsumori-Sugai, J. M. M. Caaveiro, and K. Tsumoto, J. Biol. Chem., 2010, 285, 532.

4. M. Czupryn, F. Bennett, J. Dube, K. Grant, H. Scoble, and J. M. McCoy, Ann. N. Y. Acad. Sci., 1995, 762, 152.

5. H. Yokota, M. Kishimoto, H. Saito, T. Sakao, S. Yokota, and S. Kojima, J. AOAC Int., 2000A, 83, 1053.

6. W. Zhang, M. J. Czupry, P. T. Boyle, and J. Amari, Pharm. Res., 2002, 19, 1223.

7. T. Yin, T. Taga, M. L. Tsang, K. Yasukawa, T. Kishimoto, and Y. Yang, J. Immunol., 1993, 151, 2555.

8. H. Tan, G. Dan, H. Gong, and L. Cao, Biotechnol. Lett., 2005, 27, 905.

9. M. Saitoh, K. Taguchi, K. Momose, K. Suga, Y. Ogawa, S. Yasuda, and K. Miyata, Cytokine, 2001, 13, 287.

10. W. Wang, Int. J. Pharm., 1999, 185, 129.

11. P. Lara-Quintanar, I. Lacunza, J. Sanz, J. C. Diez-Masa, and M. de Frutos, J. Chromatogr., A, 2007, 1153, 227.

12. R. A. Kenley and W. W. Nicolas, Pharm. Res., 1994, 11, 72.

13. J. V. Amari and I. Mazsaroff, J. Chromatogr., A, 1996, 728, 113. 
14. H. Yokota, H. Saito, K. Masuoka, H. Kaniwa, and T. Shibanuma, J. Pharm. Biomed. Anal., 2000B, 24, 317.

15. W. Garzon-Rodriguez, R. L. Koval, S. Chongprasert, S. Krishnan, T. W. Randolph, N. W. Warne, and J. F. Carpenter, J. Pharm. Sci., 2004, 93, 684.

16. H. Lee, J. Liq. Chromatogr. Relat. Technol., 2005, 28, 1161.

17. A. F. Bristow and S. L. Jeffcoate, Biologicals, 1992, 20, 221.

18. S. L. Dalmora, F. B. D'Avila, L. M. da Silva, A. C. Bergamo, and E. S. Zimmermann, J. Chromatogr., B, 2009, 877, 2471.

19. B. E. Almeida, J. E. Oliveira, R. Damiani, S. L. Dalmora, P. Bartolini, and M. T. C. P. Ribela, J. Pharm. Biomed. Anal., 2011, 54, 681.

20. British Pharmacopoeia, The Stationery Office, 2010, London.

21. ICH, "Guideline on Validation of Analytical Procedure: Text and Methodology Q2(R1)", in Proceedings of the International Conference on Harmonization of Technical Requirements for Registration of Pharmaceuticals for
Human Use, 2005, http://www.ich.org.

22. ICH, "Guideline on Specifications: Test Procedures and Acceptance Criteria for Biotechnological/Biological Products Q6B", in Proceedings of the International Conference on Harmonization of Technical Requirements for Registration of Pharmaceuticals for Human Use, 1999, http://www.ich.org.

23. D. R. Nogueira, M. S. Sangoi, L. M. Silva, V. Todeschini, and S. L. Dalmora, J. Sep. Sci., 2008, 31, 3098.

24. G. A. Shabir, W. L. Lough, S. A. Arain, and T. K. Bradshaw, J. Liq. Chromatogr. Relat. Technol., 2007, 30, 311.

25. A. Takagi, N. Yamashita, T. Yoshioka, Y. Takaishi, K. Nakanishi, S. Takemura, A. Maeda, K. Saito, Y. Takamura, and M. Hashida, J. Controlled Release, 2006, 115, 134.

26. G. B. Kresse, Eur. J. Pharm. Biopharm., 2009, 72, 479.

27. A. Takagi, N. Yamashita, T. Yoshioka, Y. Takaishi, K. Sano, H. Yamaguchi, A. Maeda, K. Saito, Y. Takakura, and M. Hashida, J. Controlled Release, 2007, 119, 271.

28. A. S. Groot and D. W. Scott, Trends Immunol., 2007, 28, 482. 